EDITORIAL

\title{
Untangling the Vascular Web from Alzheimer Disease and Oxidative Stress
}

Can. J. Neurol. Sci. 2012; 39: 4

Vascular abnormalities, hypoperfusion, oxidative stress, amyloid- $\beta$, and Alzheimer disease (AD) are part of an ever increasing web of confusion where causality and defining features are frequently interchanged. That we have at least two well-subscribed biannual international symposia on just the vascular element with no resolution of the connection to AD testifies to the interest and lack of resolution. Why has this come to pass? Separation of vascular changes, which are intimately linked to $\mathrm{AD}$, from "mainstream" research in $\mathrm{AD}$ is artificial and based on pre-conception. This situation arose because about 30 years ago, what is now AD was often called 'hardening of the arteries' until the AD revolution reclassified all. While significant cellular, molecular, and genetic insights into the etiology of $\mathrm{AD}$ were brought about by a precise designation, it also removed what we think of as $\mathrm{AD}$ from the concurrent vascular pathology. The inherent conflict has come full circle with the latest diagnostic criteria relying on further detached biomarkers for changes; there, the biological response is poorly understood.

The most perplexing biomarker change, amyloid- $\beta$ decrease with the progression of $\mathrm{AD}$, is still unexplained. The often cited idea that it represents amyloid- $\beta$ deposition does not bear up under scrutiny since senile plaques represent but a small percentage of the brain's amyloid- $\beta$ production. The decrease in amyloid- $\beta$ with disease progression parallels oxidative damage, which is highest at disease onset, and drops to control levels in advanced $\mathrm{AD}^{1,2}$. However, senile plaque formation is not associated with increased oxidative stress, placing in further doubt that senile plaques serve as an amyloid- $\beta$ sink. Alzheimer disease is a persistent state, one in which at least some diseaserelated biomarkers decrease while dementia increases. Could it be that these markers lack causality? Certainly the great number of abnormalities and persistence of patients with AD support this view.

The study of Shi and coworkers in this issue ${ }^{3}$ adds to the data supporting the continuity of vascular dementia with AD. The study demonstrated increased oxidative stress in patients with concurrent vascular insufficiency and dementia, but not in patients with vascular abnormalities alone. As in AD, cognitive decline defines the transition to oxidative stress (Nunomura et al, unpublished data). Also, as with $\mathrm{AD}$, oxidative markers decrease with vascular disease progression. Clearly more detailed analysis of the transition to dementia in patients suffering vascular abnormalities is required. Dementia is the common final destination to multiple interconnected paths that should not be artificially separated. Understanding the continuum between AD and vascular dementia is at the heart of the greatest public health issue before us: metabolic syndrome and consequent increased diabetes and heart disease that lead to vascular decline. Current and proposed diagnostic criteria define $\mathrm{AD}$ as a discrete condition, while evidence supports its continuity with the complex metabolic changes our body undergoes during aging and in adapting to modern lifestyles. Dissecting these issues is essential to progress in understanding and treating $\mathrm{AD}$ and vascular dementia.

George Perry, Clyde F. Phelix

University of Texas at San Antonio, San Antonio, Texas, USA

Akihiko Nunomura

University of Yamanashi, Chuo, Yamanashi, Japan

Luis V. Colom

University of Texas at Brownsville/Texas Southmost College, Brownsville, Texas, USA

Rudy J. Castellani

University of Maryland, Baltimore, Maryland, USA

Robert B. Petersen, Hyoung-gon Lee, Xiongwei Zhu Case Western Reserve University, Cleveland, Ohio, USA

\section{REFERENCES}

1. Nunomura A, Perry G, Aliev G, et al. Oxidative damage is the earliest event in Alzheimer disease. J Neuropathol Exp Neurol. 2001;60:759-67.

2. Nunomura A, Tamaoki $\mathrm{T}$, Motohashi $\mathrm{N}$, et al. Neuronal RNA oxidation during aging process and in preclinical Alzheimer disease and mild cognitive impairment. J Neuropathol Exp Neurol. In press.

3. Shi G-X, Liu C-Z, Wang L-P, Guan L-P, Li S-Q. Biomarkers of oxidative stress in vascular dementia patients. Can J Neurol Sci. 2012;39:65-8. 